Jörg-Wemer Kremp · Inmitten gehen wir nebenher 
Jörg-Wemer Kremp

\section{Inmitten gehen wir nebenher}

Nicolas Bom: Biographie, Bibliographie, Interpretationen

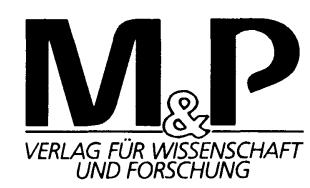


Der Abdruck der Radienung von Uwe Bremer aus der Reihe "Stadtschreiber von Bergen - Graphische Blätter", hg. von der Berger Bücherstube, erfolgt mit freundlicher Genehmigung des Künstlers und von Frau Monika Steinkopf

Die Deutsche Bibliothek - CIP-Einheitsaufnahme

\section{Kremp, Jörg-Werner:}

Inmitten gehen wir nebenher : Nicolas Born: Biographie, Bibliographie, Interpretationen / Jörg-Werner Kremp. -

Stuttgart : M und P, Verl. für Wiss. und Forschung, 1994

Zugl.: Münster (Westfalen), Univ., Diss., 1993

ISBN 978-3-476-45052-4

ISBN 978-3-476-45052-4

ISBN 978-3-476-04209-5 (eBook)

DOI 10.1007/978-3-476-04209-5

Dieses Werk ist einschließlich aller seiner Teile geschützt. Jede Verwertung außerhalb der engen Grenzen des Urheberrechtsgesetzes ist ohne Zustimmung des Verlages unzulässig und strafbar. Das gilt insbesondere für Vervielfältigungen, Übersetzungen, Mikroverfilmungen und Einspeicherung in elektronischen Systemen.

M \& P Verlag für Wissenschaft und Forschung ein Verlag der J. B.Metzlerschen Verlagsbuchhandlung und Carl Emst Poeschel Verlag GmbH in Stuttgart

(C) 1994 Springer-Verlag GmbH Deutschland Ursprünglich erschienen bei J.B. Metzlersche Verlagsbuchhandlung und Carl Ernst Poeschel Verlag GmbH in Stuttgart 1994 


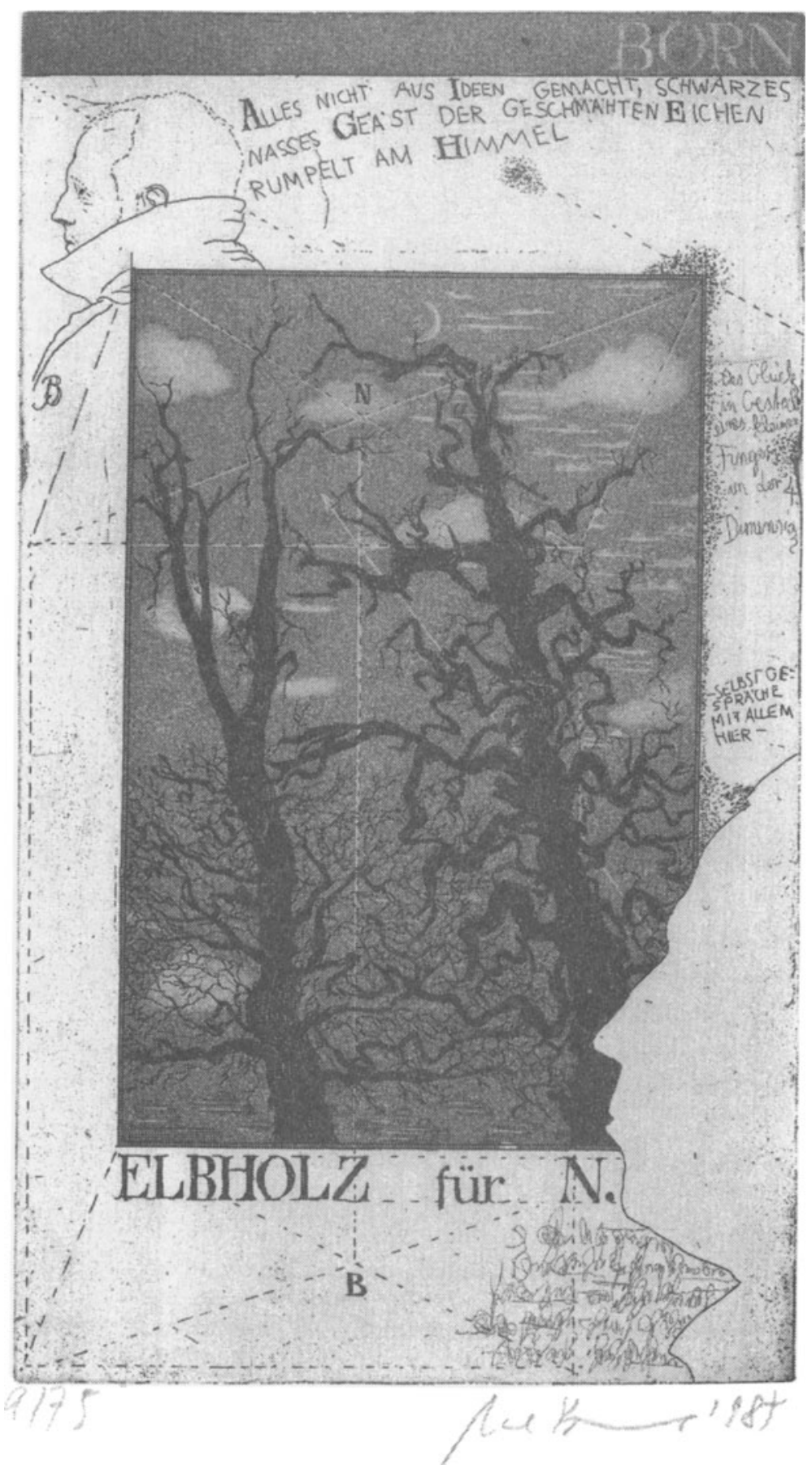




\section{Vorbemerkung}

Ohne die Hilfe anderer wäre diese Arbeit so nicht entstanden:

Prof. Dr. Ernst Ribbat hat die Auseinandersetzung mit dem Werk von Nicolas Born angeregt und auf eine in der heutigen Universitätslandschaft ungewöhnlich aufmerksame und fördernde Weise begleitet.

Die Schmittmann-Wahlen-Stiftung (Köln) ermöglichte dank der Vermittlung von Dr. P. Adenauer in den letzten beiden Jahren die intensive Beschäftigung mit dem Thema.

Der Stifter des Nicolas-Born-Preises, Dr. Hubert Burda, und seine Mitarbeiterin, Frau Dr. Christa Maar, haben durch einen Druckkostenzuschuß zur Publikation dieser Dissertation beigetragen.

Frau Monika Steinkopf, in ihrer Bücherstube Anlaufstelle für die Stadtschreiber in Bergen-Enkheim, gab mir erste Hinweise auf Freunde und Bekannte von Nicolas Born, die zu einem vielfältigen und weitreichenden Briefwechsel, teuren Telefongesprächen und spannenden Besuchen geführt haben. Allen voran $\mathrm{zu}$ danken habe ich Frau Dr. Irmgard Born für ihre geduldige Erinnerungsarbeit, ferner Undine Born-Marner, Christa Nett und Uwe Bremer sowie den vielen mit Nicolas Born befreundeten Autoren, Herausgebern und Redakteuren, die Zeit für Auskünfte gefunden haben.

Nicht abzugelten ist die Zumutung des Entzugs von Lebenszeit all jenen, die mit mir den Widerspruch zwischen Schreiben und Leben ausgehalten und mich unterstützt haben.

Und nur mit Vorwitz ist den Leserinnen und Lesern zu danken, die dieses Buch dem unablässigen Strom der unerhörten Monologe entreißen. 


\section{Inhaltsverzeichnis}

$1 \quad$ Einführung 13

$1.1 \quad \mathrm{Zu}$ Werkumfang und Textbestand 15

$1.2 \quad \mathrm{Zu}$ Rezeption und Forschungsstand 22

Der Forschungsstand $\quad 27$

1.3 Zielsetzungen 35

2 Exkurs: Zur autobiographischen Lesart der Gegenwartsliteratur

$3 \quad$ Lyrik und Prosa bis 1967

3.1 Lyrische Anfänge unter dem Einfluß von Ernst Meister

3.2 Vom Tonfall des Existentiellen zur lakonischen Bestandsaufnahme

3.3 Frühe Prosa im Kontext der Poetik der Wahrnehmung 81

3.3.1 Weiterführung der Todesthematik 83

3.3.2 Die erschriebene Wirklichkeit der Heimatstadt 93

$\begin{array}{lll}3.4 & \text { Zwischenbilanz } & 111\end{array}$

3.5 Der zweite Tag: Destruktion präformierter Wahrnehmung 118

$\begin{array}{ll}\text { 3.5.1 Die Zugfahrt als Modell der Erinnerung } & 118\end{array}$

$\begin{array}{ll}\text { 3.5.2 Die Zugfahrt als Modell auflösbarer Kontinuität } & 120\end{array}$

3.5.3 Kein Reiseroman - Destruktion eines Genres 123

3.5.4 Kein Ziel - Vom richtigen Sehen 126 
3.5.5 Kein Erleben - Mögliche Rollen 128

3.5.6 Konstruktion und Destruktion 139

Das Problem der Reihenfolge 139

Das Problem des Zusammenhangs $\quad 140$

Das Problem der Wiederholung 141

Die Unmöglichkeit von Tatsachenermittlungen 143

Die Vorführung von Klischees 144

Defizitäre Erzählhaltung und Mehrfacherzählen 148

Der Gestus des "Sich Vergewisserns" 150

Sprache 152

Beispiel Körperwahrnehmung 155

Konstruktion und Destruktion: Eine Parabel 157

3.5.7 Der zweite Tag. Ein Fazit 159

3.6 Der schriftstellerische Beginn im Überblick 165

4 "Geisterhaftes Dabeisein".

Die erdabgewandte Seite der Geschichte 167

$\begin{array}{lll}4.1 & \text { Verbindungslinien zum Frühwerk } & 171\end{array}$

4.2 Der Erzählgegenstand und seine Tradition 173

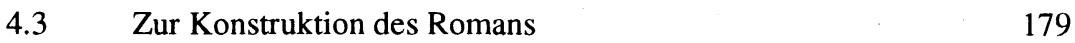

$\begin{array}{lll}\text { 4.3.1 Schreiben I } & 180\end{array}$

$\begin{array}{ll}\text { 4.3.2 Die Zeitstruktur } & 181\end{array}$

$\begin{array}{lll}\text { 4.3.3 Eine Fortsetzungsgeschichte } & 188\end{array}$

$\begin{array}{llr}4.4 & 189\end{array}$

$\begin{array}{ll}\text { 4.4.1 Das Verschwinden des Erzählers } & 192\end{array}$

$\begin{array}{ll}\text { 4.4.2 Körperwahrnehmungen } & 194\end{array}$

4.4.3 Todesmotivik, Gewalt, Sexualität, Angst 197

Die Motive Luft und Leere 201

Die psychologische Interpretationsebene 202

4.5 Die Wahrnehmung der vermittelten Welt 208

4.5.1 Fülle - Ordnung - Zusammenhang - Reihenfolge 209

$\begin{array}{ll}\text { 4.5.2 Die Aversion gegen "Erklärungen" } & 217\end{array}$

$\begin{array}{ll}\text { 4.5.2.1 Welterklärungssysteme } & 217\end{array}$

4.5.2.2 Erklärung des einzelnen 220

$\begin{array}{lll}\text { 4.5.3 Die Vermittlung durch Zeichen. Schreiben II } & 221\end{array}$

$\begin{array}{lll}\text { 4.5.4 Die Welt als Theater } & 225\end{array}$ 
4.5.5 Der einzelne und die Absage an Identität 227

$\begin{array}{ll}\text { 4.5.5.1 Das Individualgespenst } & 228\end{array}$

4.5.5.2 Das Kollektivgespenst 236

4.5.6 "Die Welt der Maschine" 239

4.5.7 Der Preis bewußter Wahrnehmung 241

4.5.8 Die Oppositionen in der vermittelten Welt 244

4.6 "Schwache Bilder einer anderen Welt" 246

4.6.1 Einzelne Glücksmomente 248

4.6.2 Ambivalenz aus Prinzip. Responsionsstrukturen 251

4.6.3 Die Flucht in die Natur 255

4.6.3.1 Das Verschwinden der einzelnen $\quad 255$

4.6.3.2 Das Ich vor der Rest-Natur 258

$\begin{array}{lll}\text { 4.6.3.3 Re-poetisierung der Welt } & 265\end{array}$

$\begin{array}{lll}\text { 4.6.4 Andere Bilder vom Ich } & 267\end{array}$

$\begin{array}{lll}4.7 & \text { Zusammenfassung } & 276\end{array}$

$5 \quad$ "Dabeisein ohne Dasein". Die Fälschung 285

5.1 Eine doppelte Geschichte 287

5.2 Material und Vorlagen 292

5.3 Beirut als Gemeinplatz 298

5.4 Die Zeitstruktur 301

5.5 Die Erzählperspektive 303

5.6 Der Beobachter, der Fremde 305

$\begin{array}{lll}5.7 & \text { Die Figuren } & 310\end{array}$

$\begin{array}{lll}5.8 & \text { Zutritt zur Wirklichkeit? } & 314\end{array}$

5.8.1 Die Unwirklichkeit einer Tat 314

5.8.2 Die Wirklichkeit in der Schrift? 321

5.8.2.1 Die fälschende journalistische Weltbehauptung 323

$5.9 \quad$ "Endspiel zu Lebzeiten" 335

5.10 Die Fälschung und die Entwicklung der Prosa 337 
6.1 Registratur des Faktischen

6.2 Die Imagination des Möglichen 351

6.3 Protest und Melancholie

8.1 Zur Biographie von Nicolas Born

8.2 Literaturverzeichnis

A. Werkverzeichnis von Nicolas Born

B. Literatur zu Nicolas Born

C. Weitere Literatur 